\title{
Status of the design of the ITER ECE diagnostic
}

\author{
G. Taylor ${ }^{1, \text { a }}$, M. E. Austin ${ }^{2}$, J. H. Beno ${ }^{3}$, S. Danani ${ }^{4}$, R. F. Ellis ${ }^{5}$, R. Feder ${ }^{1}$, J. L. Hesler ${ }^{6}$, A. E. Hubbard ${ }^{7}$, \\ D. W. Johnson ${ }^{1}$, R. Kumar ${ }^{4}$, S. Kumar ${ }^{4}$, V. Kumar ${ }^{4}$, A. Ouroua ${ }^{3}$, H. K. B. Pandya ${ }^{4}$, P. E. Phillips ${ }^{2}$, C. Roman ${ }^{1}$, \\ W. L. Rowan ${ }^{2}$, V. Udintsev ${ }^{8}$, G. Vayakis ${ }^{8}$, and M. Walsh ${ }^{8}$ \\ ${ }^{1}$ Princeton Plasma Physics Laboratory, Princeton, NJ 08543, USA \\ ${ }^{2}$ Institute for Fusion Studies, University of Texas at Austin, TX 78712, USA \\ ${ }^{3}$ Center for Electromechanics, University of Texas at Austin, TX 78758, USA \\ ${ }^{4}$ ITER-India/Institute for Plasma Research, Bhat 382428, Gandhinagar, India \\ ${ }^{5}$ University of Maryland, College Park, MD 20742, USA \\ ${ }^{6}$ Virginia Diodes, Inc. Charlottesville, VA 22902, USA \\ ${ }^{7}$ Plasma Science and Fusion Center, MIT, Cambridge, MA 02139, USA \\ ${ }^{8}$ ITER Organization, Route de Vinon sur Verdon, 13115, St Paul Lez Durance, France
}

\begin{abstract}
The baseline design for the ITER electron cyclotron emission (ECE) diagnostic has entered the detailed preliminary design phase. Two plasma views are planned, a radial view and an oblique view that is sensitive to distortions in the electron momentum distribution near the average thermal momentum. Both views provide high spatial resolution electron temperature profiles when the momentum distribution remains Maxwellian. The ECE diagnostic system consists of the front-end optics, including two $1000 \mathrm{~K}$ calibration sources, in equatorial port plug EP9, the $70-1000 \mathrm{GHz}$ transmission system from the front-end to the diagnostics hall, and the ECE instrumentation in the diagnostics hall. The baseline ECE instrumentation will include two Michelson interferometers that can simultaneously measure ordinary and extraordinary mode ECE from 70 to $1000 \mathrm{GHz}$, and two heterodyne radiometer systems, covering 122-230 GHz and 244-355 GHz. Significant design challenges include 1) developing highly-reliable $1000 \mathrm{~K}$ calibration sources and the associated shutters/mirrors, 2) providing compliant couplings between the front-end optics and the polarization splitter box that accommodate displacements of the vacuum vessel during plasma operations and bake out, 3) protecting components from damage due to stray ECH radiation and other intense millimeter wave emission and 4) providing the low-loss broadband transmission system.
\end{abstract}

\section{Introduction}

The ITER electron cyclotron emission (ECE) diagnostic will provide high spatial and temporal resolution measurements of the electron temperature profile evolution. These measurements are essential for successful completion of the ITER mission. ITER plasmas will be dominated by electron heating, in particular by alpha particle heating of electrons during the important deuterium-tritium phase of the mission. ECE electron temperature data will be important for evaluating the radial deposition profile and time evolution of the alpha particle heating. Furthermore, real-time ECE measurements will be used to identify magnetohydrodynamic (MHD) modes, such as neoclassical tearing modes that can often be deleterious to plasma performance. The real-time ECE data will provide feedback signals that guide the steering of the electron cyclotron heating $(\mathrm{ECH})$ launchers in order to suppress these deleterious MHD modes. ECE radiation will play a major role in plasma energy transport in ITER [1]. For ITER plasma scenarios where the central electron temperature, $T_{e}(0) \geq 35 \mathrm{keV}$ [2] power loss due to ECE is expected to exceed the loss due to bremsstrahlung, and electron cyclotron radiation transport will be comparable to, or even exceed, the plasma energy transport. Also, a relatively small population of fast electrons with energies above the bulk of the electron thermal distribution can modify and enhance the ECE power loss [3]. The ITER ECE diagnostic system will be used to detect the presence of non-thermal electrons and to measure the loss of power from the plasma via ECE radiation.

The front-end of the ECE diagnostic will be located in the central diagnostics shield module (DSM) in equatorial port plug 9 (EP9) (Fig. 1). The baseline design of the ITER ECE diagnostic system has entered the detailed preliminary design phase that will continue until a preliminary system design review in 2016.

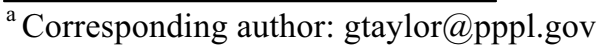




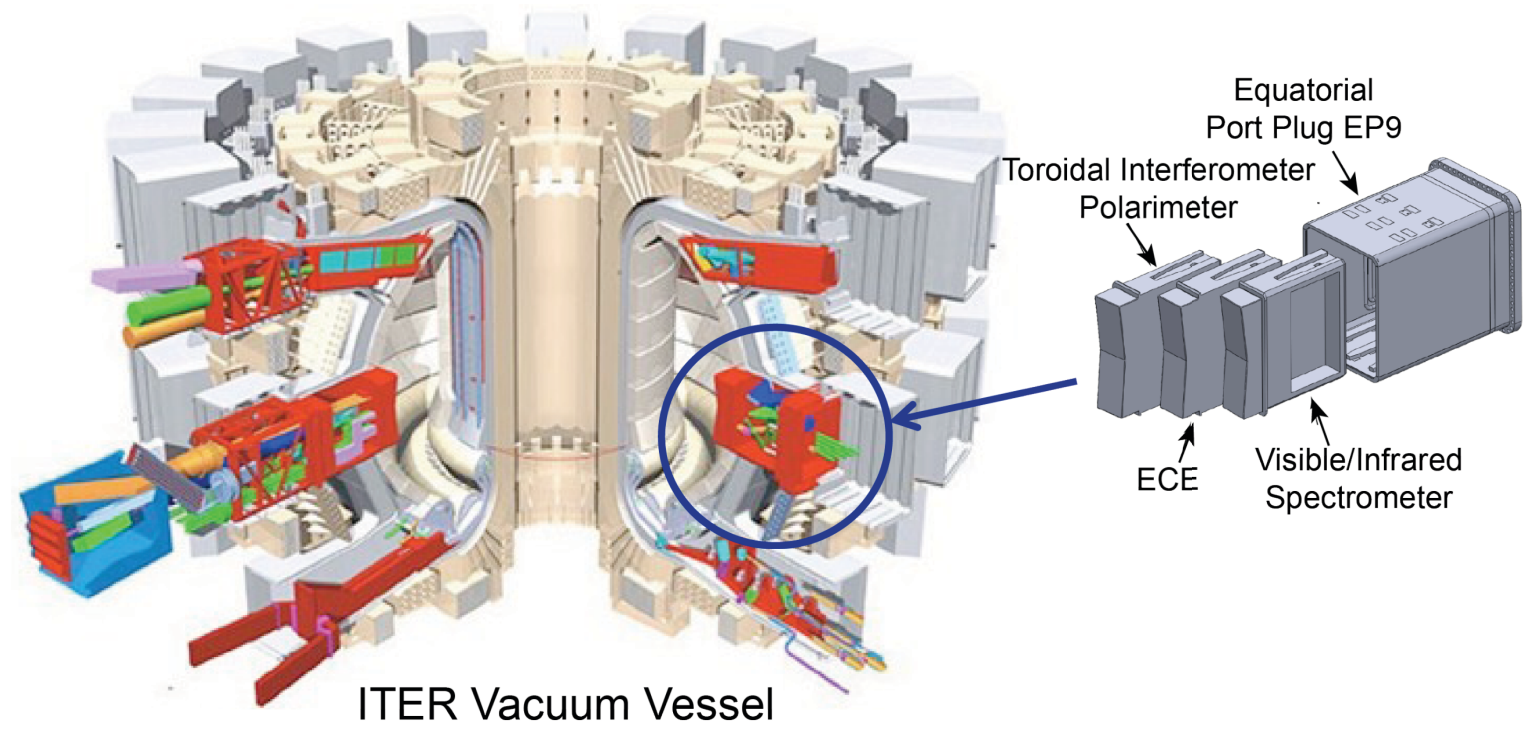

Figure 1. The ECE diagnostic will be installed in the central diagnostic shield module (DSM) of the ITER EP9 equatorial port plug.

Two plasma views are currently being planned, a radial and a moderately oblique view that is sensitive to distortions in the electron momentum distribution near the average thermal momentum [4]. There has been growing evidence that in some tokamak plasmas (eg. TFTR and JET) the electron momentum distribution can become non-Maxwellian near the average electron thermal momentum when the electron temperature exceeds $7-10 \mathrm{keV}$. Both views can provide high spatial resolution temperature profiles if the electron momentum distribution remains Maxwellian. The ECE diagnostic system can be divided into three main parts; 1) the frontend optics, including the two in-situ $1000 \mathrm{~K}$ calibration sources, in EP9, 2) the polarization splitter boxes and broadband transmission system that transports the ECE from the front-end and distributes it to the ECE instrumentation room in the diagnostics hall, and 3) the ECE instruments in the diagnostics hall. The baseline ECE instrumentation will include two Michelson interferometer systems that will simultaneously measure ordinary and extraordinary mode polarized ECE over a broad spectral range from $70 \mathrm{GHz}$ to $1 \mathrm{THz}$, and two heterodyne radiometer systems, covering 122-230 GHz and 244-355 GHz. The US Domestic Agency (US-DA) will provide the front-end components, the $244-355 \mathrm{GHz}$ radiometer system and the instrumentation in the diagnostics hall that controls and monitors the calibration sources and the associated shutters/mirrors in the frontend. The India Domestic Agency (IN-DA) will provide the remaining components of the ECE system. The control, data acquisition and analysis software will be developed jointly by the US-DA and IN-DA. The US-DA is responsible for integrating the front-end of ECE system into EP9 and the ITER organization is responsible for external interfaces, for example with buildings. Review of the final design is expected in August 2016 and will be split into several final design reviews covering components in EP9, the port cell and galleries, and the diagnostics hall. Significant design challenges include 1) developing reliable $1000 \mathrm{~K}$ calibration sources and the associated remotely controlled shutters/mirrors in EP9, 2) providing compliant couplings in the millimeter wave transmission lines between the front-end optics and the polarization splitter box that can accommodate displacements of the vacuum vessel associated with thermal expansion during plasma operations and bake out, and during plasma disruptions, 3) protecting the ECE diagnostic components from stray $\mathrm{ECH}$ radiation and other intense millimeter wave emission, and 4) providing a low-loss broadband transmission system between the front-end and the diagnostics hall. This paper reviews the current design of the baseline ECE diagnostic, design challenges that will need to be addressed during the preliminary and final design phases, and possible future upgrades to state-of-the-art ECE instrumentation. Additional information on engineering aspects of the design of the ECE diagnostic and its integration into ITER can be found in the paper by Udintsev et al. [5]. Section 2 covers the design of the optical components in the port plug and the calibration sources, Section 3 covers the design of the double vacuum window, polarization splitter boxes and low-loss, broadband transmission line, and Section 4 discusses the instrumentation in the diagnostics hall, including possible upgrades to state-ofthe-art instrumentation.

\section{Design of the front-end components}

\subsection{Front-end optical components}

The current design of the ECE diagnostic front-end (Fig. 2) has a "radial" view that views normal to the plasma outer surface and an "oblique" view that views the plasma at $13^{\circ}$ from normal. This "oblique" view 
measures ECE from higher energy electrons than the "radial" view [4]. Each view includes a $1000 \mathrm{~K}$ calibration source inside the port plug. The calibration source will be switched into each view by a remotelycontrolled mirror/shutter. The front-end design presented allowing insufficient space for shielding. Recently a decision was made to make all the DSMs the same width, as a result the current component design in the DSM will definitely not be compatible with the reduced DSM width. It will be necessary to reduce the angle of the

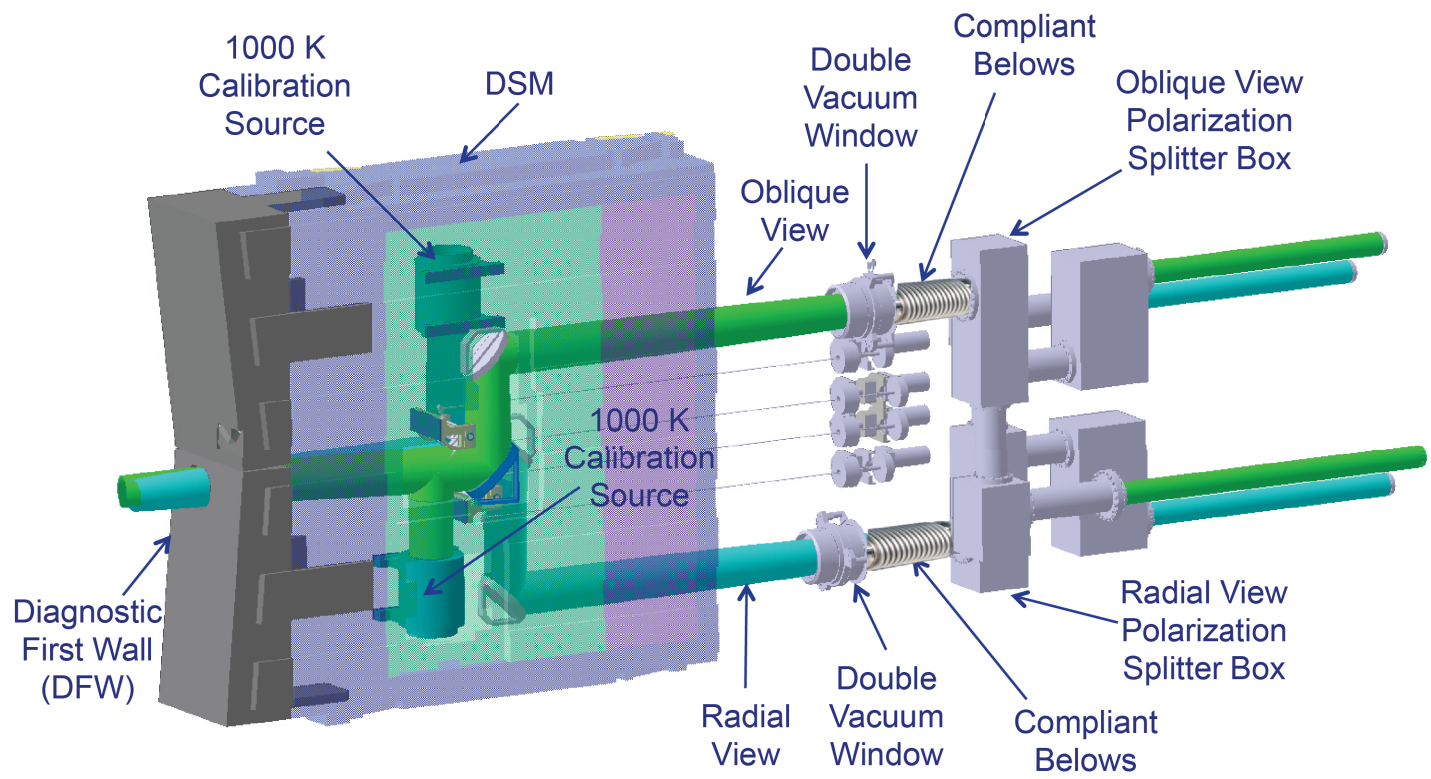

Figure 2. Layout of the ECE diagnostic components in the central DSM of the EP9 port plug and the polarization splitters mounted behind the port plug.

at the EC-17 workshop in 2012 [6] had the calibration sources positioned behind the port plug to improve access oblique view by several degrees, possibly to $\sim 10^{\circ}$ in order to have an oblique view compatible with the width

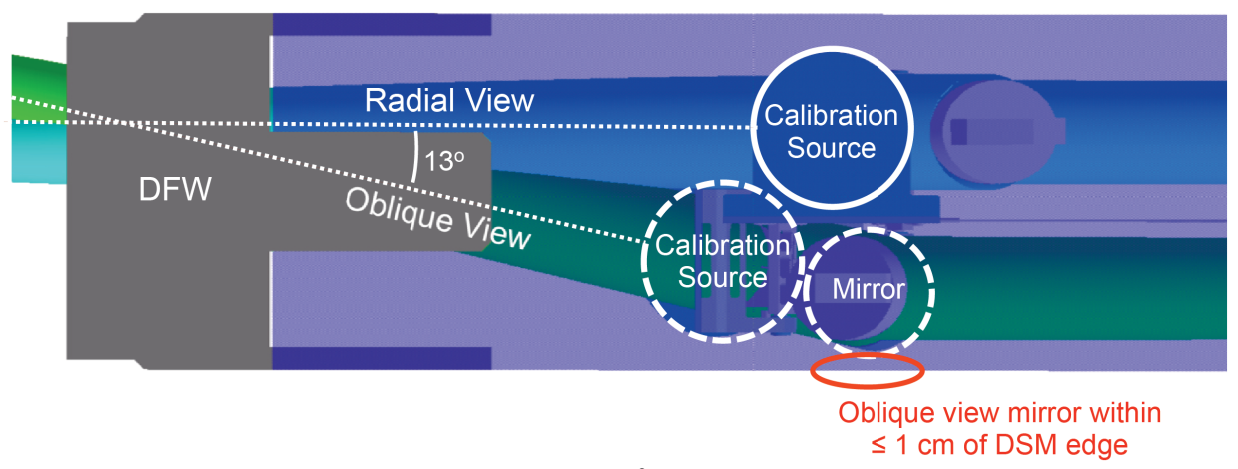

Figure 3. Top view of the ECE DSM in EP9 showing that for a $13^{\circ}$ oblique view there is little or no shield for the first mirror in the oblique view.

for maintenance. That design had additional penetrations that increased the neutron streaming rate above the level allowed on ITER.

A redesign of the front-end optics to reduce component overcrowding and improve the neutronics was completed in 2013 [7]. This new design has the calibrations sources located in the port plug close to the first mirror in each view. As a result of this redesign the required diameter of the emissive surface of the calibration sources was reduced from 20 to $15 \mathrm{~cm}$, and the sampling volume in the plasma was not changed.

The central DSM in which the ECE diagnostic frontend is located has until recently been several centimeters wider than the two adjacent DSMs. However even with this additional width the $13^{\circ}$ oblique view components are very close to the outer edge of the DSM (Fig. 3),

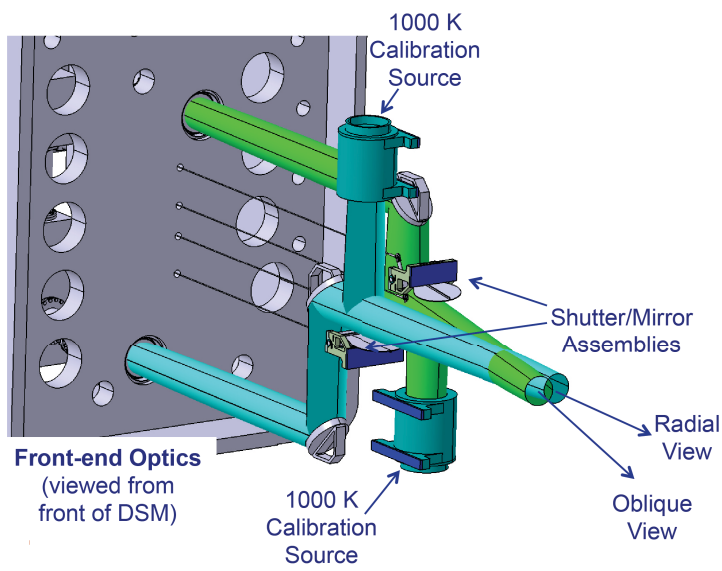

Figure 4. Front-end optics viewed from the front of the DSM showing calibration sources and shutter/mirrors. 
of the DSM and in order to meet the shielding requirements. A $10^{\circ}$ oblique view will be sensitive to electron energies of 5-20 keV, and it will still be most sensitive to electrons with momenta around the average thermal momentum.

The front-end optics design employs quasi-optical, Gaussian beams with no waveguides in the port plug. The design has remotely-actuated shutter/mirrors that will switch the hot calibration sources into the oblique and radial views (Fig. 4). These shutter/mirrors need to be highly reliable and compatible with electromagnetic, thermal and mechanical loads associated with an unmitigated plasma disruption. A 2-wire design connecting two linear actuators to each shutter/mirror is currently being evaluated. While this design mitigates the effect of port plug twisting under high disruption loads concerns have been raised about the designs compatibility with remote handling. A detailed analysis of electromagnetic and thermal loads on the mirror/shutter assembly and associated actuators is currently underway and there is a plan to test a prototype mirror/shutter assembly under ultra high vacuum conditions and during $200 \mathrm{C}$ bake cycles.

\subsection{Hot calibration sources}

(a)

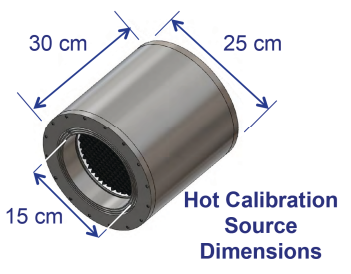

(b)

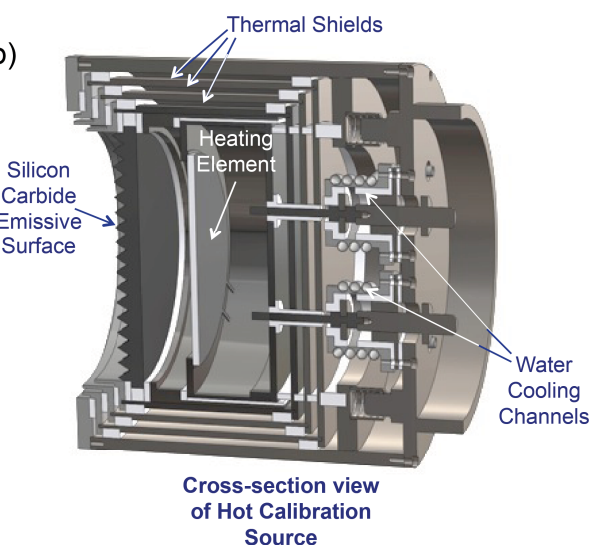

Figure 5. (a) Hot calibration source dimensions and (b) cross-section view of a prototype hot calibration source that will use a heating module with elements coated in either pyrolytic graphite or pyrolytic boron nitride.

Early prototypes of hot calibration sources that operate in vacuum and use a silicon carbide emissive surface consisting of an array of small pyramids are being tested on the J-TEXT and EAST plasma devices in China. These early prototypes use filament heaters, and while the filament heaters worked well enough to test the emissive surface and to perform testing of a nested cylinder insulation approach, the design is probably not sufficiently robust for use on ITER. Some of the free-standing heaters designs showed issues with wire sagging. This was likely due to localized wire overheating. A post-mortem analysis will be conducted on these failed heaters to better characterize the problem. The ITER calibration sources are required at achieve a nominal radiation temperature of $1000 \mathrm{~K}$ from the emissive surface with a radiation temperature accuracy of $\pm 10 \mathrm{~K}$ and a radiation temperature stability of $\pm 3 \mathrm{~K}$ over 24 hours. Most challenging is the requirement that the calibration source should operate reliably in the port plug for up to 100 calibrations, implying an operating lifetime of $>5000$ hours. Future prototype development will explore the use of commercial encapsulated heaters that have heating elements coated in either pyrolytic graphite or boron nitride or that are constructed from solid silicon carbide (Fig. 5). As mentioned earlier the diameter of the emissive surface of the calibration source was reduced from 20 to $15 \mathrm{~cm}$ through adjustments in the optics in order to be compatible with geometrical constraints in the DSM. The external diameter was reduced from 30 to 25 $\mathrm{cm}$ (Fig. 5(a)). The reduced hot source diameter will likely require a major re-design of the heater element, shields, supports, and cooling. Simple scaling of components from the earlier conceptual design is not likely to be adequate and thermal management will certainly become more challenging.

\section{Design of components between the front-end and the diagnostics hall}

\subsection{Vacuum window assembly and compliant coupling}

At the back of the port plug there will be a double crystal quartz vacuum window (Fig. 6(a)). Both windows will have a $130 \mathrm{~mm}$ diameter clear aperture. The windows will be wedged and angled to avoid interference between surfaces. The window assembles will

(b)
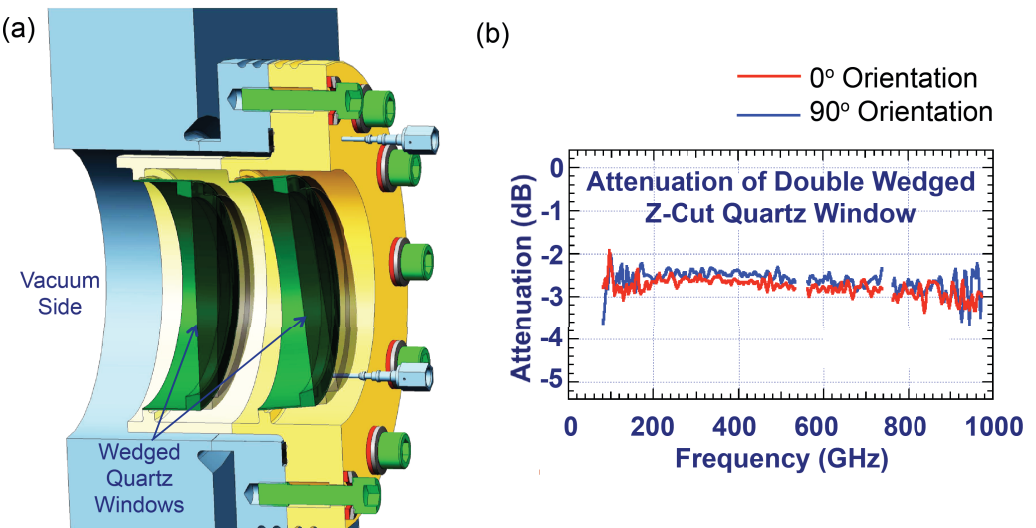

Figure 6. (a) Conceptual design for ITER double wedged crystal quartz window and (b) DIII-D Michelson interferometer transmission measurements for a double wedged crystal quartz window. 
be provided by ITER but the design will be guided by input from the US-DA and IN-DA. A bolted design is planned for better attachment to the compliant bellows that attaches between the window assemblies and the polarization splitter boxes. As the preliminary design phase moves forward it will be essential to model and mock-up transmission through the whole assembly to evaluate the effect of reflections.

Transmission measurements of a double z-cut crystal quartz window, consisting of two $4^{\circ}$ wedged windows on $15 \mathrm{~cm}$ diameter conflate flanges, using a Michelson interferometer at DIII-D, confirmed the expected flat and low transmission loss $(<3 \mathrm{~dB})$ from 80 to $1000 \mathrm{GHz}$ (Fig. 6(b)).

There will be significant radial displacement of the vacuum vessel associated with thermal expansion during plasma operations, consequently it will be necessary to install compliant bellows in the millimeter wave transmission lines between the vacuum window assemblies (Fig. 2) and the polarization splitter boxes (Fig. 7) to accommodate this displacement. Alignment of the optics will be performed with the front-end heated to the temperature expected during normal plasma operations in order to compensate for the displacement during plasma operations. The bellows will also serve to decouple displacements during bake out and, to some extent, during plasma disruptions, although they will not effectively decouple lateral displacements associated with disruptions.

\subsection{Polarization splitters}

Each polarization splitter box consists of two Gaussian beam telescopes constructed from three parabolic mirrors and one flat mirror (Fig. 7). A wire grid separates the $\mathrm{O}$ beam diameter in the front-end and increasing neutron shielding to an acceptable level. An axial tilt of $0.1^{\circ}$ increases the power lost to conversion by $\leq 1 \%$. The calculated transmission efficiency of the splitter box is $>$ 93\% from 100 to $1000 \mathrm{GHz}$. The insider surfaces of the beam splitter will be coated with absorber to minimize scattering of radiation.

\subsection{Transmission line}

Performance requirements for the broadband transmission system present a significant design challenge, the required transmission loss $\sim 15 \mathrm{~dB}$ from 70 to $400 \mathrm{GHz}$ and $22 \mathrm{~dB}$ from 400 to $1000 \mathrm{GHz}$. There will be four 45-meter long transmission lines, with an internal diameter of $72 \mathrm{~mm}$, between the EP9 port plug and the diagnostics hall, carrying X-mode and O-mode polarized ECE from the radial and oblique plasma views. The current design has 42 meters of straight waveguide in each transmission line, 10 miter bends in each of the radial view transmission lines and 11 miter bends in each of the oblique view transmission lines. A waveguide connection unit with power splitters will be installed in the ECE instrumentation area to guide ECE to the Michelson interferometers and radiometer systems.

\section{Instrumentation in the diagnostics hall}

\subsection{Baseline instrumentation}

The baseline instrumentation will include two, high throughput, reciprocating Martin-Puplett Fourier Transform Spectrometers with a spectral range of
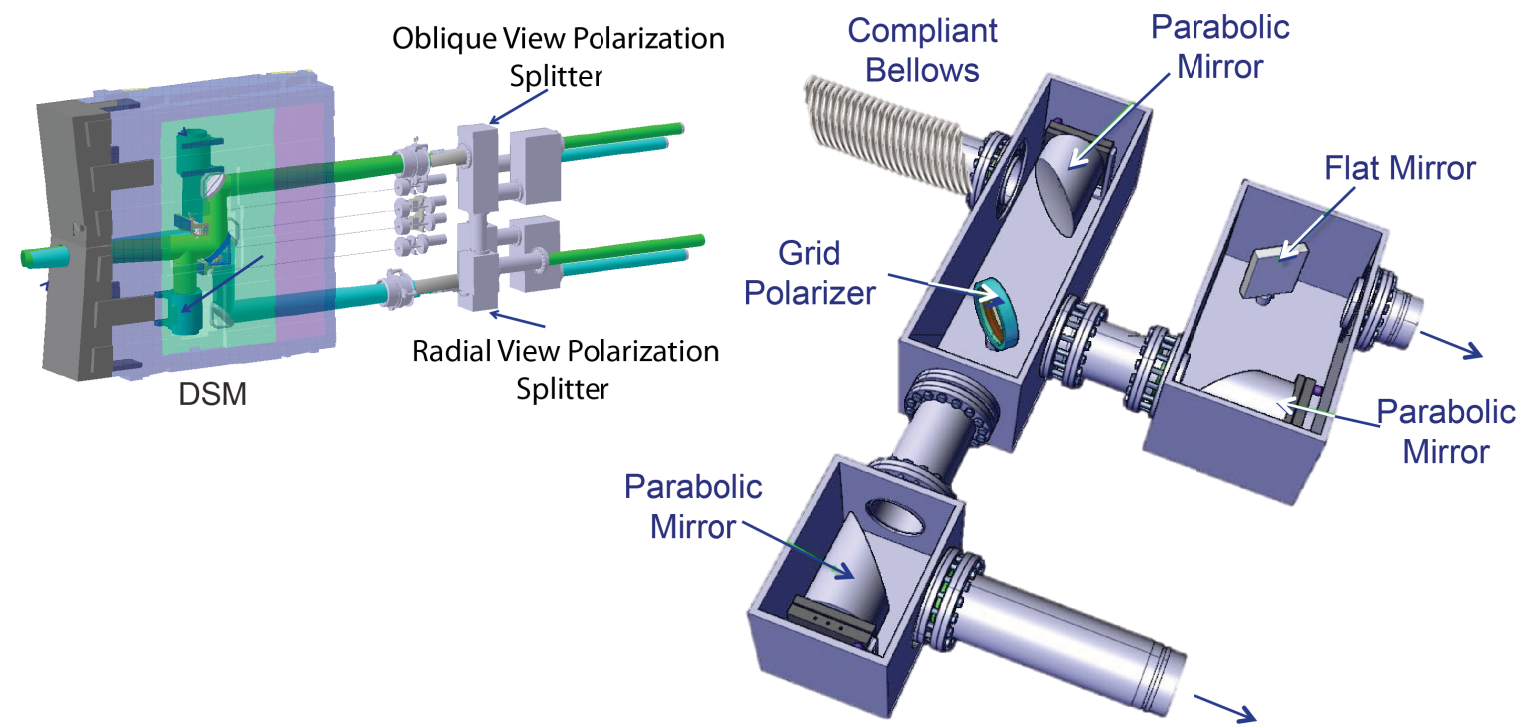

Figure 7. Polarization splitter box design is shown on the right. The location of the splitter boxes for the radial and oblique views relative to the ECE DSM is shown in the upper left.

and $\mathrm{X}$ mode polarized emission. This Gaussian telescope design allows a fixed aperture waveguide to be mapped to a variable aperture at the interface between the internal and external optics. This was key to reducing
$70-1000 \mathrm{GHz}$, a frequency resolution $\leq 3.75 \mathrm{GHz}$ and a scanning repetition rate $\leq 20 \mathrm{~ms}$. The ECE community has a lot of experience with these instruments, however they have a significant dead time between scans. Above 
$500 \mathrm{GHz}$ water vapour absorption will be significant and simply flushing with dry nitrogen will not entirely mitigate the absorption, so the broadband instruments will need to operate in vacuum. An ECE Michelson interferometer on C-Mod at MIT [8] has operated reliably in vacuum in the $100-1000 \mathrm{GHz}$ range, with a stable calibration, for many years. One possible option for the broadband instruments might be a large-throughput rotating Michelson, these have much less dead time between scans, but current versions have relatively low throughput. Another option could be a Mach-Zehnder interferometer, these are currently used widely by the sub-millimeter wave astronomy community. Finally measurements based on a Hilbert-transform spectral analysis of output from a high- $\mathrm{T}_{\mathrm{c}}$ Josephson junction detector offer the possibility of much reduced calibration time below $350 \mathrm{GHz}$, a video bandwidth of $100 \mathrm{kHz}$ and acquisition of a full spectrum in $10 \mathrm{~ms}$ [9].

The baseline instrumentation includes a set of heterodyne radiometers covering $122-355 \mathrm{GHz}$ ECE spectra and that have a video bandwidth of $500 \mathrm{kHz}$. The

\subsection{Possible upgrades to "State-of-the-Art" instrumentation}

Upgrades to the baseline instrumentation that allow increased capability and flexibility of the ECE diagnostic system need to be considered. Recent rapid advances in the speed of digital circuitry have allowed direct digitization of intermediate frequency (IF) signals in the $\mathrm{GHz}$ frequency regime and even microwave signals up to $30 \mathrm{GHz}$ and beyond. Development of software-defined radios has been progressing well into the $\mathrm{GHz}$ frequency regime. We can expect these advances to continue throughout the 20-30 year lifespan of ITER.

Microwave emission measurements on TEXTOR used Fast Fourier Transform processing of IF signals to acquire $4 \mathrm{GHz}$ wide spectra in the range $136-140 \mathrm{GHz}$ [10]. Post-processing of the digitized data allowed the number of spectra per second, and corresponding frequency resolution $(\Delta \mathrm{f})$, to be varied from 120 spectra/sec $(\Delta \mathrm{f}=25 \mathrm{kHz})$ to $200,000 \mathrm{spectra} / \mathrm{s}$ $(\Delta \mathrm{f}=100 \mathrm{MHz})$. Near-real time processing of data and flexible reconfiguration of the data acquisition system is

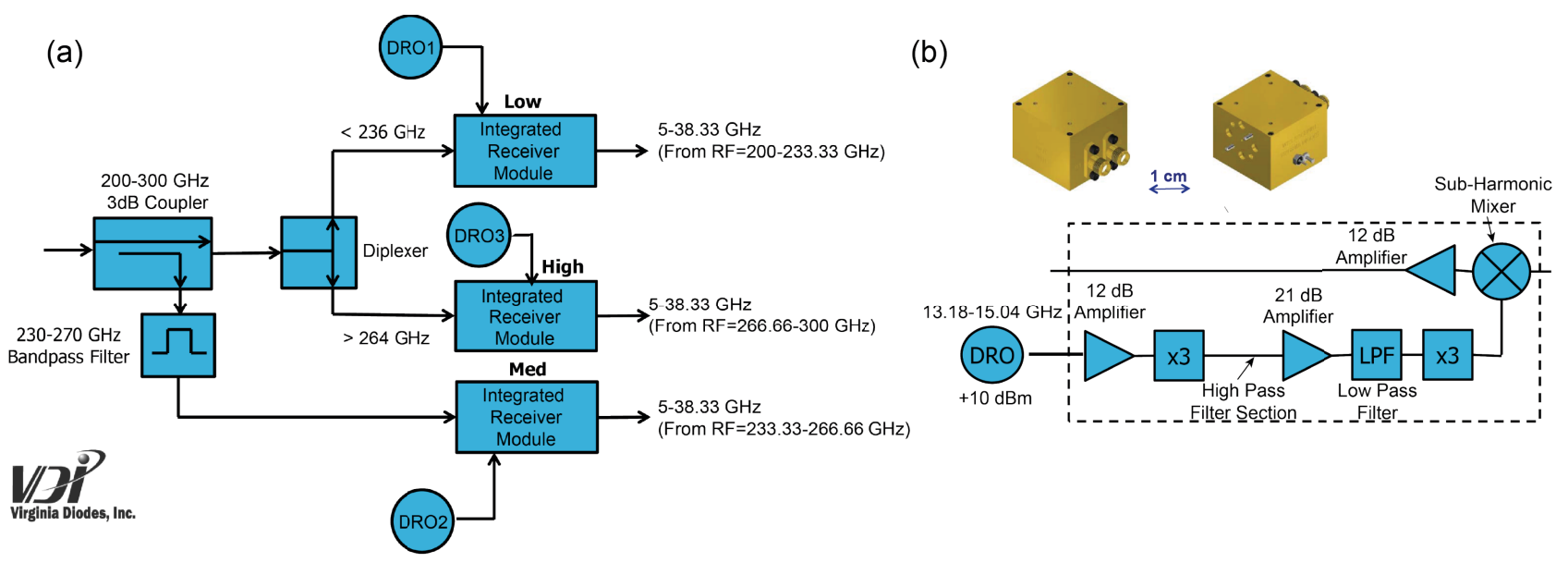

Figure 8. (a) Schematic of the prototype 200-300 GHz receiver front-end being developed by Virgina Diodes Inc. (b) Schematic of components in the integrated $5-38.33 \mathrm{GHz}$ receiver module.

IN-DA will provide a $122-230 \mathrm{GHz}$ radiometer system. That system is currently planned to include the following receivers: a $122-138 \mathrm{GHz}$ receiver with sixteen $1 \mathrm{GHz}$ bandwidth channels, a $138-168 \mathrm{GHz}$ receiver with sixteen $2 \mathrm{GHz}$ bandwidth channels, a $172-218 \mathrm{GHz}$ receiver with sixteen $3 \mathrm{GHz}$ bandwidth channels, and a $220-230 \mathrm{GHz}$ receiver with three $4 \mathrm{GHz}$ bandwidth channels.

The US-DA will provide a $244-355 \mathrm{GHz}$ radiometer system. The current plan is to provide fortyeight $2 \mathrm{GHz}$ bandwidth channels and sixteen highresolution channels with a bandwidth of $250 \mathrm{MHz}$. The US-DA is considering using a highly integrated front-end design for the $244-355 \mathrm{GHz}$ radiometer based on a 200 - $300 \mathrm{GHz}$ prototype receiver currently being developed by Virginia Diodes in the US (Fig. 8(a)). This receiver uses three identical integrated receiver modules (Fig. 8(b)). made possible by using Field Programmable Gate Array and Graphics Processing Unit architectures in these IF data acquisition systems.

Analog to digital convertors (ADCs) constructed from Josephson junction comparators have been used to sample frequencies up to $30 \mathrm{GHz}$ in superconducting integrated circuits (ICs) operating at a temperature of $4 \mathrm{~K}$. These ADCs are connected to rapid single flux quantum digital "channelizer" circuits located on the same IC to form a digital microwave receiver [11]. It may be possible to extend this technology to higher microwave frequencies in the near future.

\section{Summary}

ECE measurements will be essential for the successful completion of the ITER mission. Detailed design of the 
ITER ECE diagnostic system is now underway in a collaborative effort between India and the US. The design retains a radial and oblique plasma view and in-situ calibration sources in the front-end. Several design challenges remain to be addressed during the preliminary design phase. Advances in digital and microwave technology promise to provide an opportunity to make significant upgrades to the baseline ECE instruments during the lifetime of ITER.

The views and opinions expressed herein do not necessarily reflect those of the ITER Organization.

\section{References}

1. F. Albajar, et al., Nucl. Fusion, 45, 642 (2005)

2. V. Parail, et al., Nucl. Fusion 53, 113002 (2013)

3. K. V. Cherepanov and A. B. Kukushkin, Proc. 20th Int. Conf. Fusion Energy, Vilamoura, Portugal, November 1-6, 2004, paper TH0P6-56, IAEA (2004)

4. G. Taylor and R. W. Harvey, Fus. Sci, Tech. 55, 64 (2009)

5. V. S. Udinstev, et al, "Engineering aspects of design and integration of ECE diagnostic in ITER", Proc. $18^{\text {th }}$ Joint Workshop on Electron Cyclotron Emission and Electron Cyclotron Heating, Nara, Japan (2014)

6. M. E. Austin, et al., Proc. $17^{\text {th }}$ Joint Workshop on Electron Cyclotron Emission and Electron Cyclotron Heating, Deurne, The Netherlands, EPJ Web of Conferences 32, 03003 (2012)

7. W. L. Rowan, et. al. "ITER ECE Front End Optical Design Modifications", Final Report PPPL S012583-F

8. A. E. Hubbard, T. C. Hsu, and P. J. O'Shea, Proc. $9^{\text {th }}$ Joint Workshop on Electron Cyclotron Emission and Electron Cyclotron Heating, Borrego Springs, California, p. 387, World Scientific (1995)

9. H. K. B. Pandya and Y. Divin, "New approach to ECE measurements based on Hilbert-transform spectral analysis", Proc. $18^{\text {th }}$ Joint Workshop on Electron Cyclotron Emission and Electron Cyclotron Heating, Nara, Japan (2014)

10. D. J. Thoen, et al. Rev. Sci. Instrum. 80, 103504 (2009)

11. D. E. Kirichenko, et al. Microwave Symposium Digest MTT 09 pp. 1449-52 (2009) 